\title{
PROJECTIVE REPRESENTATIONS OF QUIVERS
}

\section{SANGWON PARK}

Received 30 August 2001

We prove that $P_{1} \stackrel{f}{\rightarrow} P_{2}$ is a projective representation of a quiver $Q=\bullet \rightarrow \bullet$ if and only if $P_{1}$ and $P_{2}$ are projective left $R$-modules, $f$ is an injection, and $f\left(P_{1}\right) \subset P_{2}$ is a summand. Then, we generalize the result so that a representation $M_{1} \stackrel{f_{1}}{\longrightarrow} M_{2} \stackrel{f_{2}}{\longrightarrow} \cdots \stackrel{f_{n-2}}{\longrightarrow} M_{n-1} \stackrel{f_{n-1}}{\longrightarrow}$ $M_{n}$ of a quiver $Q=\bullet \rightarrow \bullet \rightarrow \cdots \bullet \rightarrow \bullet \rightarrow \bullet$ is projective representation if and only if each $M_{i}$ is a projective left $R$-module and the representation is a direct sum of projective representations.

2000 Mathematics Subject Classification: 16E30, 13C11, 16D80.

1. Introduction. A quiver is just a directed graph. We allow multiply edges and edges going from a vertex to the same vector. Originally a representation of a quiver assigned a vertex space to each vertex and a linear map to each edge (or arrow) with the linear map going from the vector space assigned to the initial vertex to the one assigned to the terminal vertex. For example, a representation of a quiver $\bullet \rightarrow \bullet$ is $V_{1} \stackrel{f}{\longrightarrow} V_{2}$. Then we can define a morphism of two representations of the same vector. Now, instead of vector spaces we can use left $R$-modules and also instead of linear maps we can use $R$-linear maps. In this paper, we study the properties of projective representations of quivers. Representations of quivers is a new topic in module theory and is recently developed in $[1,2]$.

DEFINITION 1.1. A representation $P_{1} \stackrel{f}{\longrightarrow} P_{2}$ of a quiver $Q=\bullet \rightarrow \bullet$ is said to be projective if every diagram of representations

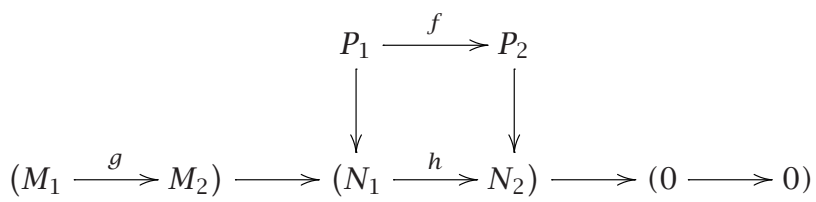

can be completed to a commutative diagram as follows:

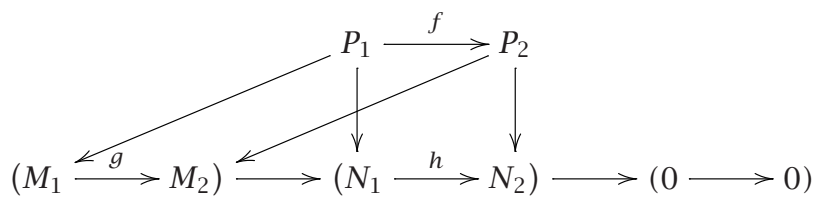

LEMMA 1.2. If $P_{1} \stackrel{f}{\longrightarrow} P_{2}$ is a projective representation of a quiver $Q=\bullet \rightarrow \bullet$, then $P_{1}$ and $P_{2}$ are projective left $R$-modules. 
Proof. Let $M$ and $N$ be left $R$-modules, $\alpha: P_{1} \rightarrow N$ an $R$-linear map, and $\beta: M \rightarrow N$ an onto $R$-linear map. Then, since $P_{1} \stackrel{f}{\longrightarrow} P_{2}$ is a projective representation, we can complete the diagram

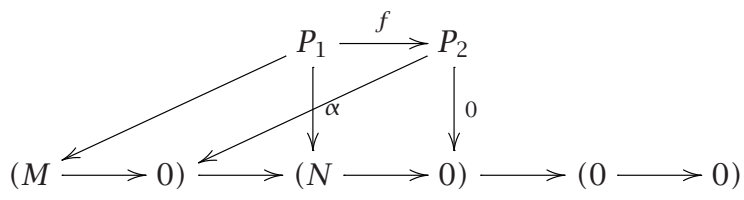

as a commutative diagram. Thus $P_{1}$ is a projective left $R$-module.

Let $g: P_{2} \rightarrow N$ be an $R$-linear map and let $h: M \rightarrow N$ be an onto $R$-linear map. Then, since $P_{1} \stackrel{f}{\longrightarrow} P_{2}$ is a projective representation, we can complete the diagram

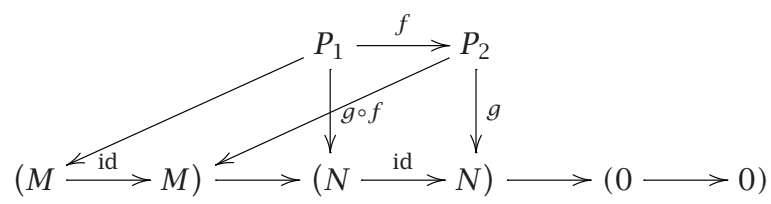

as a commutative diagram. Thus $P_{2}$ is a projective left $R$-module.

LEMMA 1.3. If $P$ is a projective left $R$-module, then a representation $0 \rightarrow P$ of a quiver $Q=\bullet \rightarrow \bullet$ is a projective representation.

Proof. The lemma follows by completing the diagram

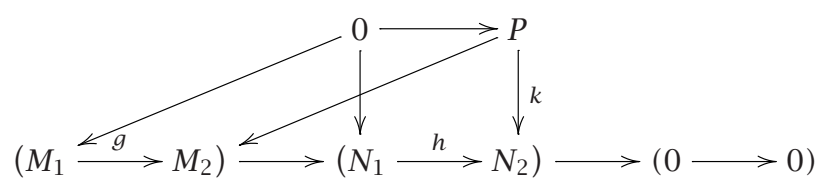

as a commutative diagram.

REMARK 1.4. A representation $P \rightarrow 0$ of a quiver $Q=\bullet \rightarrow \bullet$ is not a projective representation if $P \neq 0$, because we cannot complete the diagram

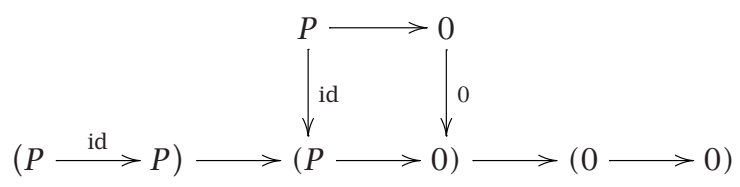

as a commutative diagram.

LEMMA 1.5. If $P$ is a projective left $R$-module, then a representation $P \stackrel{\mathrm{id}}{\longrightarrow} P$ of $a$ quiver $Q=\bullet \rightarrow \bullet$ is a projective representation. 
Proof. Let $M_{1}, M_{2}, N_{1}$, and $N_{2}$ be left $R$-modules and let $g: M_{1} \rightarrow M_{2}$ and $h: N_{1} \rightarrow$ $N_{2}$ be $R$-linear maps. Let $k: P \rightarrow N_{1}$ be an $R$-linear map and choose $h \circ k: P \rightarrow N_{2}$ as an $R$-linear map. And consider the following diagram:

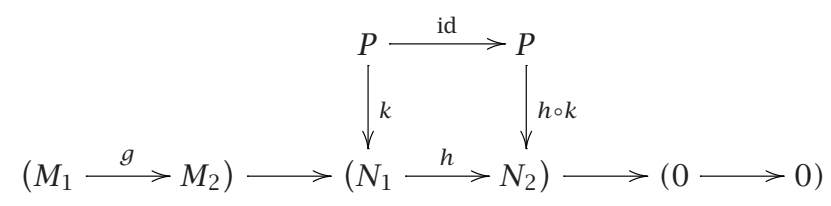

Then, since $P$ is a projective left $R$-module, there exists a map $\alpha: P \rightarrow M_{1}$. Now choose $g \circ \alpha: P \rightarrow M_{2}$ as an $R$-linear map. Then $\alpha$ and $g \circ \alpha$ complete the above diagram as a commutative diagram. Therefore $P \stackrel{\text { id }}{\longrightarrow} P$ is a projective representation.

\section{Direct sum of projective representations}

THEOREM 2.1. A representation $P_{1} \stackrel{f}{\longrightarrow} P_{2}$ of a quiver $Q=\bullet \rightarrow \bullet$ is projective if and only if $P_{1}$ and $P_{2}$ are projective left $R$-modules, $f$ is an injection, and $f\left(P_{1}\right) \subset P_{2}$ is a summand.

Proof. Consider the following diagram:

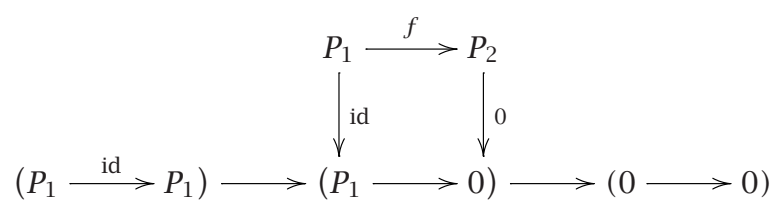

Since $P_{1} \stackrel{f}{\longrightarrow} P_{2}$ is a projective representation, we can complete the above diagram as a commutative diagram as follow:

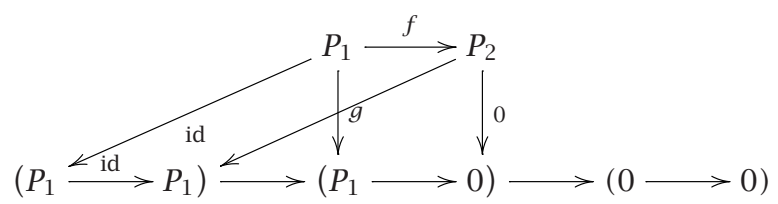

Thus $g \circ f=\operatorname{id}_{P_{1}}$. Therefore, $P_{2} \cong f\left(P_{2}\right) \oplus \operatorname{ker}(g)$ and

$$
\left(P_{1} \longrightarrow P_{2}\right) \cong\left(P_{1} \longrightarrow\left(P_{1} \oplus \operatorname{ker}(g)\right)\right) \cong\left(P_{1} \stackrel{\text { id }}{\longrightarrow} P_{1}\right) \oplus(0 \longrightarrow \operatorname{ker}(g))
$$

This completes the proof.

Now let $Q=\bullet \rightarrow \bullet \rightarrow \bullet \cdot \rightarrow \bullet \rightarrow \bullet$ be a quiver with $n$ vertices and $n-1$ arrows. Then, we can easily generalize the results of Lemmas 1.3 and 1.5 as follows: the 
representations

$$
\begin{gathered}
0 \longrightarrow 0 \longrightarrow \cdots \longrightarrow 0 \longrightarrow 0 \longrightarrow P_{n}, \\
0 \longrightarrow 0 \longrightarrow \cdots \longrightarrow 0 \longrightarrow P_{n-1} \stackrel{\text { id }}{\longrightarrow} P_{n-1}, \\
\vdots \\
0 \longrightarrow P_{2} \stackrel{\text { id }}{\longrightarrow} \cdots \rightarrow P_{2} \stackrel{\text { id }}{\longrightarrow} P_{2} \stackrel{\text { id }}{\longrightarrow} P_{2}, \\
P_{1} \stackrel{\text { id }}{\longrightarrow} P_{1} \stackrel{\text { id }}{\longrightarrow} \cdots \stackrel{\text { id }}{\longrightarrow} P_{1} \stackrel{\text { id }}{\longrightarrow} P_{1} \stackrel{\text { id }}{\longrightarrow} P_{1}
\end{gathered}
$$

are all projective representations of a quiver $Q=\bullet \rightarrow \bullet \rightarrow \bullet \cdot \bullet \rightarrow \bullet \bullet$, if each $P_{i}$ is a projective left $R$-module. We can also generalize Lemma 1.2 so that if $M_{1} \stackrel{f_{1}}{\longrightarrow}$ $M_{2} \stackrel{f_{2}}{\longrightarrow} \cdots \stackrel{f_{n-1}}{\longrightarrow} M_{n-1} \stackrel{f_{n}}{\longrightarrow} M_{n}$ of a quiver $Q=\bullet \rightarrow \bullet \rightarrow \cdots \bullet \rightarrow \bullet \bullet$ is projective representation, then each $M_{i}$ is a projective left $R$-module.

THEOREM 2.2. A representation $M_{1} \stackrel{f_{1}}{\longrightarrow} M_{2} \stackrel{f_{2}}{\longrightarrow} M_{3}$ of a quiver $Q=\bullet \rightarrow \bullet \rightarrow$ is projective if and only if $M_{1}, M_{2}$, and $M_{3}$ are projective left $R$-modules, $f_{1}\left(M_{1}\right)$ is a summand of $M_{2}$, and $f_{2}\left(M_{2}\right)$ is a summand of $M_{3}$. That is,

$$
\left(M_{1} \longrightarrow M_{2} \longrightarrow M_{3}\right) \cong\left(P_{1} \stackrel{\text { id }}{\longrightarrow} P_{1} \stackrel{\text { id }}{\longrightarrow} P_{1}\right) \oplus\left(0 \longrightarrow P_{2} \stackrel{\text { id }}{\longrightarrow} P_{2}\right) \oplus\left(0 \longrightarrow 0 \longrightarrow P_{3}\right)
$$

Proof. The diagram

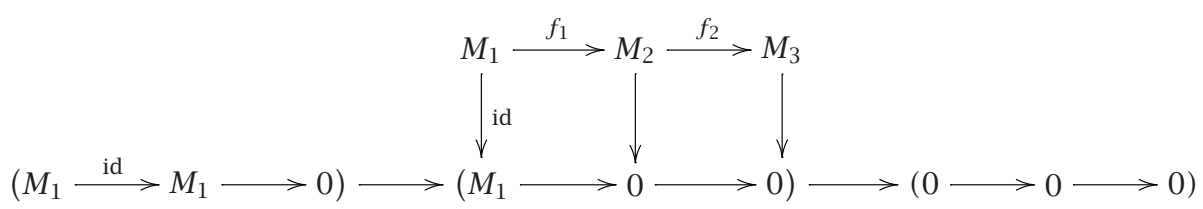

can be completed to a commutative diagram by id : $M_{1} \rightarrow M_{1}, g_{21}: M_{2} \rightarrow M_{1}$, and $0: M_{3} \rightarrow 0$. Then we can get $g_{21} \circ f_{1}=\operatorname{id}_{M_{1}}$ so that $M_{2} \cong M_{1} \oplus \operatorname{ker}\left(g_{21}\right)$. Now the diagram

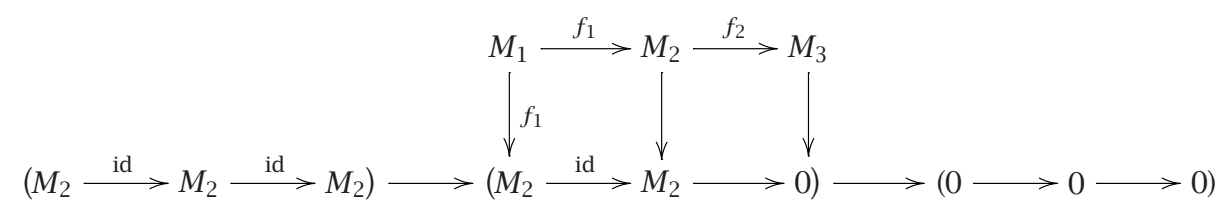

can be completed to a commutative diagram by $f_{1}: M_{1} \rightarrow M_{2}$, id $: M_{2} \rightarrow M_{2}$, and $g_{32}$ : $M_{3} \rightarrow M_{2}$. Then, we can get $g_{32} \circ f_{2}=\operatorname{id}_{M_{2}}$ so that $M_{3} \cong M_{2} \oplus \operatorname{Ker}\left(g_{32}\right)$. Therefore, $M_{3} \cong M_{2} \oplus \operatorname{Ker}\left(g_{32}\right) \cong M_{1} \oplus \operatorname{Ker}\left(g_{21}\right) \oplus \operatorname{Ker}\left(g_{32}\right)$. Hence,

$$
\left(M_{1} \longrightarrow M_{2} \longrightarrow M_{3}\right) \cong\left(P_{1} \stackrel{\text { id }}{\longrightarrow} P_{1} \stackrel{\text { id }}{\longrightarrow} P_{1}\right) \oplus\left(0 \longrightarrow P_{2} \stackrel{\text { id }}{\longrightarrow} P_{2}\right) \oplus\left(0 \longrightarrow 0 \longrightarrow P_{3}\right) .
$$

This completes the proof. 
Now, we can easily generalize Theorem 2.2 so that a representation $M_{1} \stackrel{f_{1}}{\longrightarrow} M_{2} \stackrel{f_{2}}{\longrightarrow}$ $\cdots \stackrel{f_{n-2}}{\longrightarrow} M_{n-1} \stackrel{f_{n-1}}{\longrightarrow} M_{n}$ of a quiver $Q=\bullet \rightarrow \bullet \rightarrow \bullet \cdots \bullet \bullet \rightarrow \bullet$ is projective representation if and only if each $M_{i}$ is a projective left $R$-module and the representation is the direct sum of the following projective representations:

$$
\begin{gathered}
0 \longrightarrow 0 \longrightarrow \cdots \rightarrow 0 \longrightarrow 0 \longrightarrow P_{n}, \\
0 \longrightarrow 0 \longrightarrow \cdots \rightarrow 0 \longrightarrow P_{n-1} \stackrel{\text { id }}{\longrightarrow} P_{n-1}, \\
\vdots \\
0 \longrightarrow P_{2} \stackrel{\text { id }}{\longrightarrow} \cdots \stackrel{\text { id }}{\longrightarrow} P_{2} \stackrel{\text { id }}{\longrightarrow} P_{2} \stackrel{\text { id }}{\longrightarrow} P_{2}, \\
P_{1} \stackrel{\text { id }}{\longrightarrow} P_{1} \stackrel{\text { id }}{\longrightarrow} \cdots \stackrel{\text { id }}{\longrightarrow} P_{1} \stackrel{\text { id }}{\longrightarrow} P_{1} \stackrel{\text { id }}{\longrightarrow} P_{1} .
\end{gathered}
$$

REMARK 2.3. The representations of a quiver $Q=\bullet \rightarrow \bullet \rightarrow \bullet \cdots \bullet \bullet \rightarrow \bullet$ :

$$
\begin{gathered}
P \longrightarrow 0 \longrightarrow 0 \longrightarrow \cdots \rightarrow 0 \longrightarrow 0 \\
P \stackrel{\text { id }}{\longrightarrow} P \longrightarrow 0 \longrightarrow 0 \longrightarrow \cdots \rightarrow 0 \\
\vdots \\
P \stackrel{\text { id }}{\longrightarrow} P \stackrel{\text { id }}{\longrightarrow} \cdots \stackrel{\text { id }}{\longrightarrow} P \stackrel{\text { id }}{\longrightarrow} P \longrightarrow 0
\end{gathered}
$$

are not projective representations if $P \neq 0$.

ACKNOWLEDGment. This work was supported by Korea Science and Engineering Foundation (KOSEF) 2001-1-10200-001-1.

\section{REFERENCES}

[1] E. E. Enochs and I. Herzog, A homotopy of quiver morphisms with applications to representations, Canad. J. Math. 51 (1999), no. 2, 294-308.

[2] E. E. Enochs, I. Herzog, and S. Park, Cyclic quiver rings and polycyclic-by-finite group rings, Houston J. Math. 25 (1999), no. 1, 1-13.

Sangwon Park: Department of Mathematics, Dong-A University, Pusan, 604-714, KOREA

E-mail address: swpark@mai 1.donga.ac.kr 


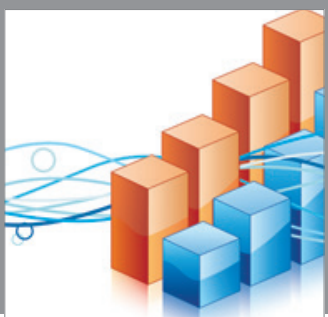

Advances in

Operations Research

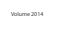

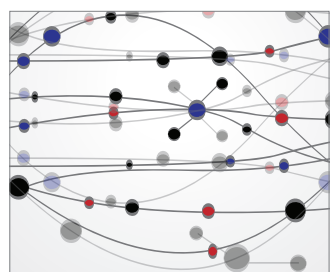

\section{The Scientific} World Journal
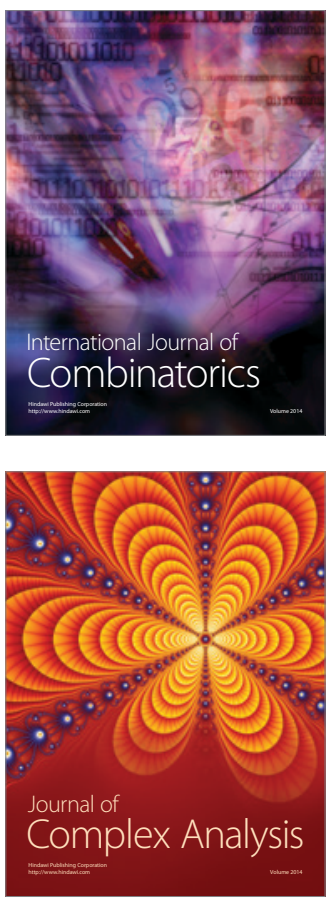

International Journal of

Mathematics and

Mathematical

Sciences
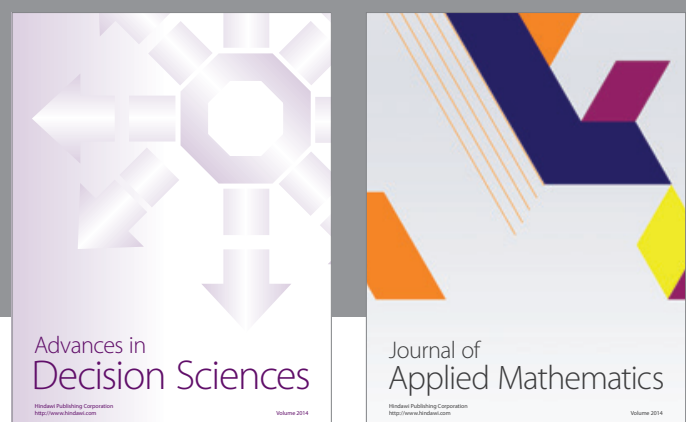

Journal of

Applied Mathematics
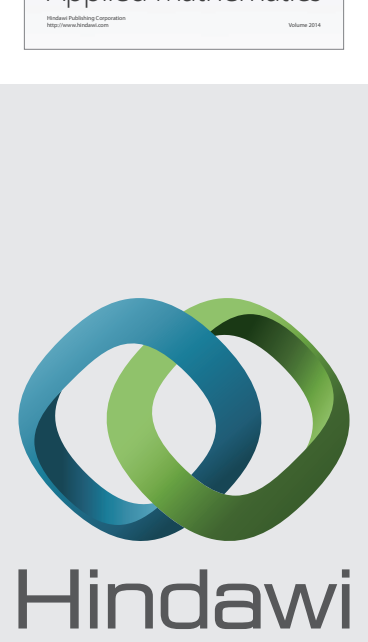

Submit your manuscripts at http://www.hindawi.com
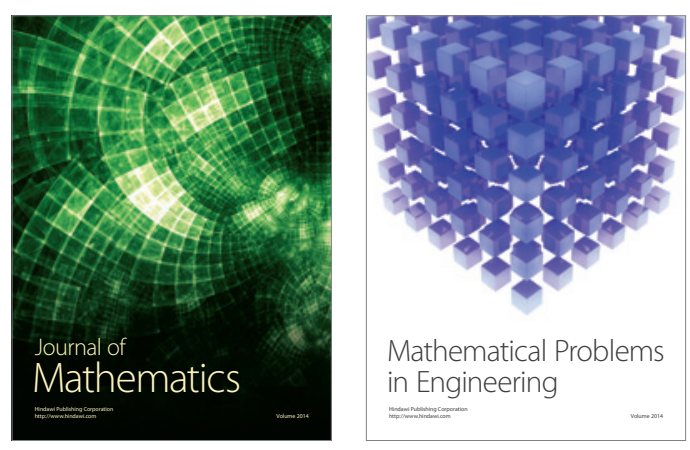

Mathematical Problems in Engineering
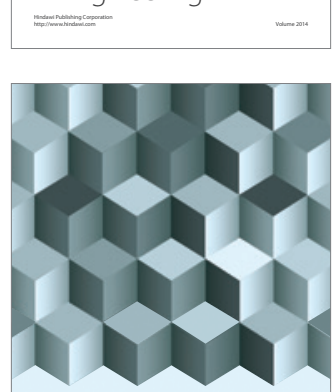

Journal of

Function Spaces
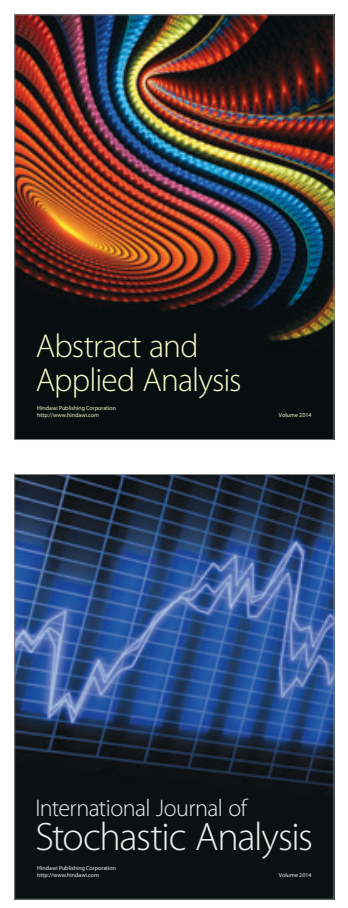

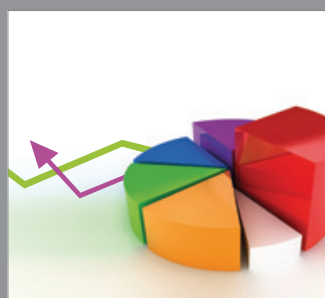

ournal of

Probability and Statistics

Promensencen
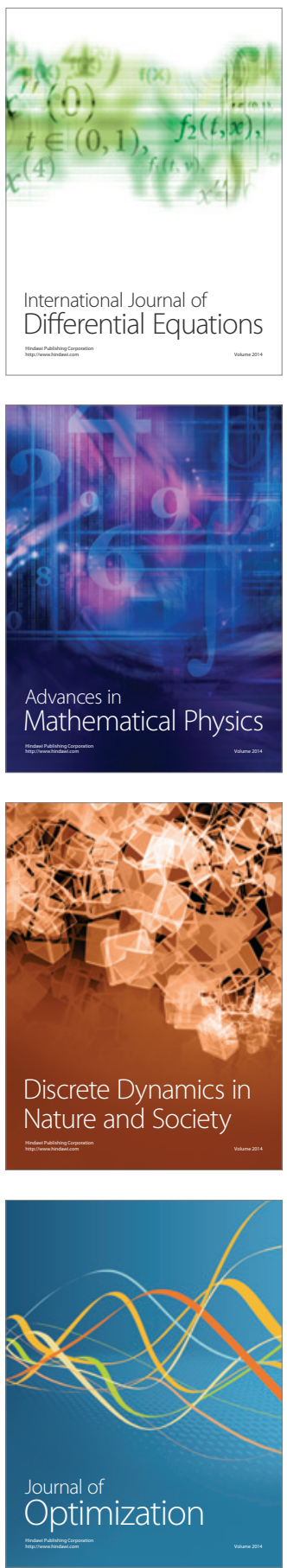